\title{
Special issue on UIT (Ubiquitous Information Technology) for multimedia systems
}

\author{
Min Hong • Vladimir Hahanov • Chen Yu
}

Published online: 24 March 2013

(C) Springer Science+Business Media New York 2013

Ubiquitous Information Technology (UIT) has rapidly emerged as a new computing paradigm and it covers the topics of seamless, secure, visible, and intuitive access to provide computing and communication services at anytime and anywhere. There are many issues to stably provide UIT services and much effort and enormous attention have been focused on the UIT environments. The UIT research area poses challenges such as context information, security, reliability, autonomous and intelligent networking, digital and multimedia systems, and so on. The UIT offers an unprecedented opportunity for various modern multimedia applications and systems under convergence of multimedia technology and multimedia visualization. Its main purpose is to solve the various problems of advanced digital and multimedia processing using the convergence of state-of-the-art computer science technology.

In UIT environments, the most important goal is to provide users more realistic and richer ubiquitous services with ultra-modern multimedia technology. In a word, we should try to guarantee and provide trustworthy and constant visual services in anyplace. Most of all, ubiquitous applications and services can be limited by data transmission, view area, and computing power. Therefore, much more attention and effort should be focused on the ubiquitous applications and services in visualization and multimedia environments. For these reasons, the proposed special issue intends to give an overview of the state-of-the-art of issues and guidelines for multimedia systems under the ubiquitous environment requiring wireless communication. In addition, it will provide completing the panorama of current research effort, which is widely inherent to topics of high interest for the computer graphics and multimedia readers.

This special issue includes three excellent papers from the selected papers of 2010 International Conference on Ubiquitous Information Technologies and Applications (CUTE 2010) and from the regular submissions of other excellent researchers related to this special issue topic. The selected papers of CUTE 2010 which were presented on December 16-18, 2010 in Sanya, China are revised and extended from the original version of conference papers. To contain

\footnotetext{
M. Hong $(\bowtie)$

Department of Computer Software Engineering, Soonchunhyang University, Asan, South Korea e-mail: minhong71@gmail.com

V. Hahanov

Kharkiv National University of Radio Electronics, 14 Lenin Avenue, 61166 Kharkov, Ukraine

C. $\mathrm{Yu}$

School of Computer Science and Technology, Huazhong University of Science and Technology, Wuhan, China
} 
high-quality papers, all papers in this special issue are carefully reviewed through a rigorous peer-review process for this SI publication.

This special issue includes the ontology-based multimedia environments for mobile devices. "Multimedia access to mobile environments using indoor semantic maps: Applications for light devices and hand-held computers" by Javier Medina et al. presents a proposal for representing the buildings and indoor spaces on the mobile devices. They designed an ontology for indoor maps, which integrates multimedia resources in the environments and users can view the location of the multimedia resources on the map and accessed them in real time using mobile devices.

"Color laser printer forensic based on noisy feature and support vector machine classifier" by Jung-Ho Choi et al. provides a new color laser printer forensics algorithm to enhance the reliability of digital images among various information in the ubiquitous space. In their experimental results show that $99.3 \%, 97.4 \%$ and $88.7 \%$ accuracy for the brand, toner and model identification respectively using 4,800 images from 8 color laser printer models where half of the image is for training and the other half is for classification.

The paper "Speech authentication by semi-fragile speech watermarking utilizing analysis by synthesis and spectral distortion optimization" by Bin Yan and Yin-Jing Guo introduces an improved semi-fragile speech watermarking scheme by quantization of linear prediction (LP) parameters. Their experimental results show that the AbS based embedding algorithm can effectively reduce the difference between the watermarked LP parameters and the extracted LP parameters hence can reduce the minimum required authentication length.

We would like to express thanks to all people who have contributed their immense time and efforts in making this successful special issue. We thank all the authors who contributed their valuable papers for this special issue. Moreover, the paper review process would not have been possible without the kind assistance of the reviewers. Finally, we would like to pay thanks to an Editor-in-Chief the Multimedia Tools and Applications, Prof. Borko Furht, and Springer staff for their encouragement and strong support during the preparation of this special issue.

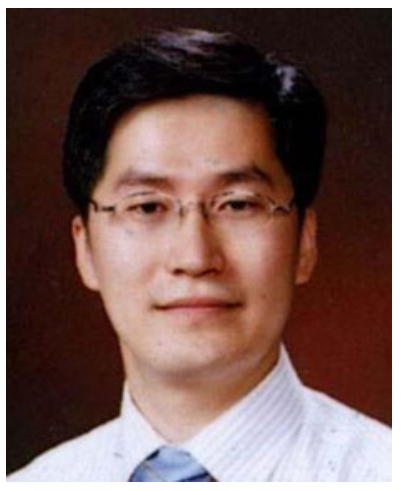

Min Hong received B.S. in Computer Science from Soonchunhyang University in 1995. He also received M.S. in Computer Science and Ph.D. in Bioinformatics from the University of Colorado in 2001 and 2005, respectively. He had worked as an instructor at the Department of Computer Science and Engineering at University of Colorado. Since 2006, he has been with the Department of Computer Software and Engineering, Soonchunhyang University, Asan, Korea, where he is an associate professor, working in the areas of Computer Graphics, Mobile Computing, Physically-based Modeling and Simulation, Bioinformatics Applications, and u-Healthcare Applications. He has published about 40 refereed professional research papers. In present, he is director of Computer 
Graphics Laboratory at Soonchunhyang University and is working on the editorial board of the TIIS (Transactions on Internet and Information Systems), JIPS (Journal of Information Processing Systems), and journal of KMMS (Korea Multimedia Society), KKITS (Korea Knowledge Information Technology Society) and KSII (Korean Society for Internet Information). He also received some funded research grants from KOSEF, KRF which are research group of Korea governments. He was also a member of steering committee of TIIS (Transactions on Internet and Information Systems), has served as workshop co-chair for ICUT 2009 (the 4th International Conference on Ubiquitous Information Technologies \& Applications) and as System Management Chair for ISPEC 2010 (The 6th Information Security Practice and Experience Conference), and many served as a Program Committee member such as ICONI/APIC-IST 2009 (The 1st International Conference on Internet \& The 4th Asia Pacific International Conference on Information Science and Technology), CGMS-09 (The 2009 International Workshop on Computer Graphics, Multimedia and Security), CSA 2009 (The 2nd International Conference on Computer Science and its Applications), APIC-IST 2008 (The 3rd Asia Pacific International Conference on Information Science and Technology) and so on.

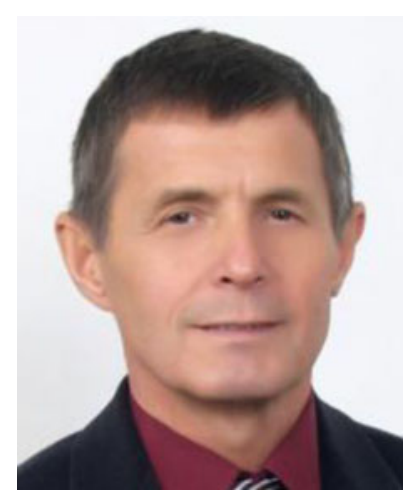

Vladimir Hahanov IEEE Senior Member, IEEE Computer Society Golden Core Member, received circuit engineer in Kharkov National University of Radio Electronics in 1978. He also received Ph.D in 1985 and Dr of Science in 1996 in Kharkov National University of Radio Electronics, Ukraine. He had worked as senior lecturer in Kharkov National University of Radio Electronics, Ukraine from 1988 up to 1997. He works as Dean of the Computer Engineering Faculty, Kharkov National University of Radio Electronics from 2003 till now, professor of Kharkov National University of Radio Electronics. Kharkov Military University, Kharkov Academy of railway transport, Kharkov Academy of Culture, Kharkov Aerospace University, Chernigov National Technical University, Lugansk National Technical University from 1997 till now. He has 450 publications, 15 books, 3 patents. He is scientific supervisor of 4 Dr of Science and $25 \mathrm{Ph}$. D's in the fields 05.13.05- "Computer systems and components", 05.13.12 "Computer Aided Design"; Supervised more than 100 Bachelor/Master degree works. He also received: 1996 -"Best methodologist of University". 2000, 2001—honors from "Best scientist of Kharkov region". 2003-INTEL award of scientific projects competition. 2005-The best professor of Ukraine. 2005-Award from President of Ukraine. 2005-IEEE Diploma for the IEEE conference organization. 2005-IEEE Computer Society Golden Core Member. 2007-IEEE Outstanding contribution Award. 2008The best dean Honor Prize of all Universities of Kharkov City. 2009-Honor Core "For Scientific Contribution". Other Activities: Chief-editor of two journals: "Radio Electronics and informatics" and "Computer aided control systems and devices". Member of three specialized scientific boards for defense of thesis for a Doctor's degree: D 64.052.02 - systems of design automation, D 64.807.02 - information technologies in control systems. Leader of the scientific seminar "Design automation and diagnosis of computational devices, systems and networks". Chairman of the international symposium "IEEE East-west. Design and Test". Member of 19 organization committee for the International Conferences. Member of IEEE Computer Society since 2000. Member of High Examination Board of Ministry of Education of Ukraine. Scientific supervisor of "Design \& Test" R\&D Lab. Chief Scientist of Aldec Inc., cooperation with Cadence, Microsoft, Intel. Scientific consultant of Echostar, USA. Hobbies: music, traveling, basketball, ski, soccer. Date of birth: $27 \mathrm{Feb} 1953$. Family status: married, four children. 


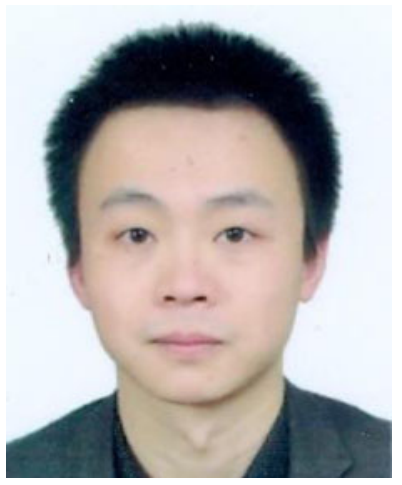

Chen Yu received his B.S. in Mathematics and M.S. in Computer Science from Wuhan University in 1998 and 2002 respectively. He received Ph.D. in Information Science from Tohoku University 2005. He had worked as a Japan Science and Technology Agency postdoctoral researcher in Japan Advanced Institute of Science and Technology from 2005 to 2006. From 2006, he had worked as a Japan Society for the Promotion of Science postdoctoral fellow in Japan Advanced Institute of Science and Technology to 2008. Since 2008, he has been with the School of Computer Science and Technology, Huazhong University of Science and Technology, China, where he is an associate professor and a special research fellow, working in the areas of Wireless Sensor Networks, Ubiquitous Computing and Green Communications. He has published more than 20 referred professional research papers and received the best paper award in ICC 2005 (the IEEE International Conference on Communication) and the nominated best paper award in DS-RT 2007 (the 11th IEEE International Symposium on Distributed Simulation and Real-Time Applications). He has served as the workshop co-chair for CIT 2009 (the 9th IEEE International Conference on Computer and Information Technology) and NPC 2010 (the 7th IFIP International Conference on Network and Parallel Computing). 